\title{
Complications of Multiple Myeloma Therapy, Part 2: Risk Reduction and Management of Venous Thromboembolism, Osteonecrosis of the Jaw, Renal Complications, and Anemia
}

\author{
Ruben Niesvizky, MD, and Ashraf Z. Badros, MD, ${ }^{\mathrm{b}}$ New York, New York, and Baltimore, Maryland
}

\section{Key Words}

Thalidomide, bortezomib, lenalidomide, deep vein thrombosis, pulmonary embolism, bisphosphonates, erythropoiesis-

stimulating agents

\begin{abstract}
Venous thromboembolism (VTE), osteonecrosis of the jaw, renal failure, and anemia are all common complications of multiple myeloma therapy. Many of these adverse events have been documented only in the past 5 to 10 years, in conjunction with the introduction of a series of the newer therapies thalidomide, bortezomib, and lenalidomide. This article discusses these complications in detail and provides strategies for health care providers to best prevent, identify, and manage them. Preventive measures, such as VTE prophylaxis and appropriate dental hygiene, as well as patient education, dose adjustments, limited duration of drug treatment, and consideration of therapies that are associated with less burdensome adverse-event profiles, can contribute to substantially improved outcomes and quality of life. (JNCCN 2010;8[Suppl 1]:S13-S20)
\end{abstract}

Therapies such as thalidomide, bortezomib, and lenalidomide have provided meaningful benefits in multiple myeloma (MM), such as improved response rates

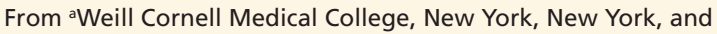
bUniversity of Maryland, Greenebaum Cancer Center, Baltimore, Maryland.

Dr. Niesvizky has disclosed that he has participated as an advisory board member, speakers' bureau member, or consultant for Celgene Corp.; Millennium Pharmaceuticals, Inc.; and Proteolix, Inc. Dr. Badros has no interest, arrangement, or affiliation with the manufacturers of any products discussed in the article or their competitors.

This work was supported by an educational grant from Millennium Pharmaceuticals, Inc.

Correspondence: Ruben Niesvizky, MD, Weill Cornell Medical College, 520 East 70th Street, Starr 341, New York, NY 10021.

E-mail: run9001@med.cornell.edu
}

and improved duration of response, but they have been associated with an increased risk of certain adverse events. Venous thromboembolism (VTE), osteonecrosis of the jaw $(\mathrm{ONJ})$, renal failure, and anemia are all common complications of MM therapy. Community oncologists should be familiar with these potential complications and the strategies for preventing, identifying, and managing them.

\section{Venous Thromboembolism}

VTE typically manifests as deep vein thrombosis (DVT) or pulmonary embolism (PE). Both cancer and cancer treatments have been identified as discrete risk factors. ${ }^{1}$ Current evidence indicates that cancer increases thrombosis risk 4.1-fold and chemotherapy increases the risk 6.5 -fold. ${ }^{2}$ Other key VTE risk factors include older age, recent surgery, acute medical illness, and immobility or bed rest. The risk of VTE is typically cumulative. ${ }^{1}$ Therefore, even if an independent relationship between VTE and specific cancers such as MM were excluded, the advanced age of most MM patients and other factors common in MM patients would tend to place them at heightened VTE risk. ${ }^{3}$ A recent, retrospective analysis of a managed care database of patients with $\mathrm{MM}(\mathrm{N}=1732)$ showed that $66.3 \%$ had at least 1 VTE risk factor and $48.3 \%$ had at least 2 risk factors. ${ }^{4}$

It has been posited that the hypercoagulable state typical with MM and other cancers provides a prothrombotic baseline environment. The prothrombotic state associated with MM may be due to multiple hemostatic abnormalities, including activation of coagulation pathways, reduced natural anticoagulation mechanisms, 
and an inflammatory milieu. ${ }^{5-8}$ Acquired resistance to activated protein $\mathrm{C}$ is a common coagulation $\mathrm{ab}$ normality in $\mathrm{MM}$, and it has also been associated with increased thromboembolism risk. ${ }^{5}$ Conditions that result in hypercoagulable states include immunoglobulin interference with fibrin structure and paraprotein acting as an autoantibody against intrinsic anticoagulants and phospholipids. ${ }^{9-11} \mathrm{~A}$ small recent study $(\mathrm{N}=49)$ of patients with $\mathrm{MM}$ receiving thalidomide confirmed that most patients who subsequently experienced a thrombotic episode had an underlying hypercoagulability abnormality. ${ }^{\text {? }}$

Underlying VTE risk often escalates with therapeutic intervention and is typically greatest after initial therapy. For example, approximately half of VTE events in patients with MM occur within 2 months of treatment initiation. ${ }^{5}$ Varying risk levels are associated with specific therapies and therapeutic combinations. In particular, VTE rates are high in patients receiving the immunomodulatory drugs thalidomide or lenalidomide in combination with highdose dexamethasone, doxorubicin, or combination chemotherapy. ${ }^{12}$ Dexamethasone alone has also been shown to somewhat increase thrombosis risk..$^{8,13,14}$

\section{Thalidomide}

A recent systematic review and meta-analysis of 17 randomized, controlled trials found an overall VTE incidence of $11.7 \%$ in thalidomide-treated patients (95\% CI, 8.1\%-16.5\%). ${ }^{15}$ This finding should be considered in the context of a systemic review by Zangari et al., ${ }^{5}$ who assessed relapsed/refractory versus newly diagnosed MM, as well as thalidomide monotherapy versus combination therapy, and found wide variation in VTE risk. Studies of thalidomide monotherapy showed VTE rates of less than $2 \%$ to $4 \%$ in both newly diagnosed patients and those with relapsed/refractory disease. Newly diagnosed patients receiving combination therapy had rates ranging from $17 \%$ to $28 \%$. The rates of VTE in patients with relapsed/refractory MM who received combination thalidomide therapy tended to be lower ( $8 \%$ to $21 \%$ ) but were still a cause for concern. The combination regimens evaluated included thalidomide/dexamethasone, thalidomide/melphalan/prednisone, and thalidomide plus conventional chemotherapy.

\section{Lenalidomide}

As with thalidomide, the risk for VTE with lenalidomide is low, ranging from $0 \%$ to less than $5 \%$, when it is administered as a single agent. ${ }^{16,17}$ However, studies have shown elevated VTE risk ranging from $8.5 \%$ to $15.0 \%$ when lenalidomide is combined with dexamethasone. ${ }^{8,14,18,19}$ More recent trials have shown that lowering the dexamethasone dosage reduces the risk of VTE with lenalidomide substantially. ${ }^{20,21}$

Heightened VTE risk has also been associated with the use of erythropoiesis-stimulating agents (ESAs), such as epoetin alfa and darbepoetin alfa. ${ }^{12}$ Anemia is discussed in more detail in subsequent sections.

\section{Bortezomib}

The proteasome inhibitor bortezomib does not appear to increase VTE risk; in fact, it may exert antithrombotic actions. Proteasome inhibition is known to decrease expression of endothelial and vascular cell adhesion molecules; in addition, bortezomib has been shown to inhibit adenosine diphosphateinduced platelet aggregation. ${ }^{22}$ Two phase III studies indicate that bortezomib is associated with thrombocytopenia rates ranging from $20 \%$ to $26 \%$ (grade 3 ) and $4 \%$ to $17 \%$ (grade 4 ), but a very low (1\%) VTE rate. ${ }^{23,24}$

\section{Treatment and Prophylaxis Recommendations}

Current VTE prophylaxis guidelines from the American College of Chest Physicians (ACCP) recommend primary antithrombotic prophylaxis for patients with cancer only if they are bedridden or undergoing surgical intervention. ${ }^{1}$ However, as discussed previously, without prophylaxis, the risk for VTE in treated MM patients is unacceptably high. In addition, PE is associated with a high case-fatality rate. One-week survival after PE is $71 \%$, but one-quarter of all cases present as sudden death. ${ }^{25}$ Therefore, identifying high-risk patients and providing them with appropriate prophylaxis is essential. Unfortunately, very few randomized studies have assessed the impact of anticoagulant prophylaxis in patients with cancer.

The International Myeloma Working Group (IMWG) recently published guidelines for preventing VTE in myeloma. ${ }^{12}$ Primary data were lacking in many cases, so the IMWG recommendations are based on expert assessment or data extrapolation from studies not designed specifically to assess prophylaxis efficacy or safety. Therefore, they should not override the treating physician's best judgment. Because VTE risk is frequently cumulative, patients 
may present with a combination of disease-related and individual risk factors, as well as inherited and non-inherited risk factors. All of these must be considered alongside therapeutically mediated risk. Table 1 lists risk factors and related screening considerations for MM patients. ${ }^{2,12,26}$

According to the IMWG, selection of candidates for thromboprophylaxis should be based on the baseline VTE risk associated with each therapy. ${ }^{12}$ The IMWG recommends reducing VTE risk to less than $10 \%$ using the safest and least cumbersome prophylaxis available. High-dose dexamethasone administration is considered an independent risk factor for VTE. At minimum, and coinciding with "black-box" warnings added recently to the prescribing information for thalidomide and lenalidomide, newly diagnosed MM patients being treated with either of those drugs in combination with dexamethasone should receive thromboprophylaxis. Proposed prophylaxis strategies include the use of a low-molecular-weight heparin such as enoxaparin, warfarin, or aspirin. Clinical experience suggests that full-dose warfarin may be used as prophylaxis, but low-dose warfarin is not acceptable. Specific IMWG recommendations are summarized in Table $2 .{ }^{12}$

For patients receiving epoetin alfa, the FDA recommends administration of an anticoagulant prophylactic. ${ }^{27,28}$ The manufacturer of darbepoetin alfa does not recommend anticoagulant prophylaxis, but it specifies that the lowest dose needed should be used to reduce thromboembolism risk. ${ }^{29}$ The combination of bortezomib with an ESA does not appear to increase the risk of VTE. ${ }^{30}$

Despite best efforts, prophylaxis cannot prevent all VTE incidents, and proper identification and management of VTE is critical. When DVT is suspected, the standard diagnostic test is compression ultrasonography. Suspected PE is typically investigated with imaging techniques, primarily CT pulmonary angiography. If this is contraindicated (due to baseline nephropathy, for example), magnetic resonance pulmonary angiography and/or nuclear medicine V/Q scan may be considered). ${ }^{12,31}$ In addition, all patients should be provided with education regarding the clinical symptoms of VTE (i.e., skin redness, pain in the extremities or chest, shortness of breath, rapid heartbeat) and instructed to inform their physician promptly if any concerns arise. If DVT is confirmed, the overall goals of treatment are
Table 1 Risk Factors to Consider When Screening Multiple Myeloma Patients for Thromboprophylaxis

Individual Factors

General patient characteristics

- Age

- Obesity and/or diabetes

- Cardiovascular or renal disease

- Acute infection

Inherited thrombophilic abnormalities

- Antithrombin III deficiencies

- Protein $\mathrm{C}$ and protein $\mathrm{S}$ deficiencies

- Acquired protein $C$ resistance

- Factor V Leiden mutation

- Prothrombin gene (G20210A) mutation

- Elevated homocysteine levels

Central venous catheter use

Prior deep vein thrombosis, pulmonary embolism, or superficial vein thrombosis

Disease-Related Factors

Recent ( $<3 \mathrm{mo}$ ) anesthesia, surgery, trauma, or hospital admission

Confinement (i.e., to a nursing home), immobilization, or sedentary lifestyle

Other malignant neoplasm, with or without chemotherapy

Hyperviscosity or other blood-clotting disorders

Neurologic disease with extremity paresis

Therapy-Related Factors

High-dose dexamethasone

Thalidomide, lenalidomide

Adjuvant doxorubicin for other cancer

Multiagent chemotherapies

Erythropoietin use

Data from Refs. 2, 12, and 26.

symptomatic relief, prevention of emboli formation, and prevention of VTE recurrence. ${ }^{12}$ Proposed DVT treatment strategies are outlined in Figure 1.

\section{Osteonecrosis of the Jaw}

A rare side effect recently documented in MM and other cancers, ONJ is linked to long-term use of bisphosphonates. The incidence of $\mathrm{ONJ}$ in bisphosphonate-treated MM patients is unknown. Manufacturer-sponsored epidemiologic studies re- 
Table 2 Recommendations for Thromboprophylaxis in Multiple Myeloma Patients Treated With Thalidomide or Lenalidomide

- $\leq 1$ VTE risk factor: aspirin (81-325 mg once daily)

- $\geq 2$ risk factors: LMWH (equivalent to enoxaparin, $40 \mathrm{mg} / \mathrm{d}$ )

- LMWH is also recommended for all patients receiving thalidomide or lenalidomide plus high-dose dexamethasone or doxorubicin

- Barring contraindications in the front-line setting, treatment strategies using dexamethasone should use low-dose dexamethasone and include aspirin prophylaxis

- Full-dose warfarin (target INR 2-3) is an alternative to LMWH, although limited data exist to support this strategy

- Anticoagulant prophylaxis is recommended in patients with relapsed disease and a high risk for VTE

- It may be reasonable to deliver anticoagulant prophylaxis for 4 to 6 months; longer treatment periods may be considered in the presence of additional risk factors

- If VTE occurs in patients receiving thalidomide or lenalidomide, it is reasonable to briefly discontinue therapy and resume once proper anticoagulation has been established

Abbreviations: INR, International Normalized Ratio; LMWH, low molecular weight heparin; VTE, venous thromboembolism. Data from Palumbo A, Rajkumar SV, Dimopoulos MA, et al. Prevention of thalidomide- and lenalidomide-associated thrombosis in myeloma. Leukemia 2008;22:414-423.

port an incidence ranging from $0.1 \%$ to $1.8 \%$. By contrast, in independent epidemiologic reports from clinicians and the International Myeloma Foundation, the incidence estimates are higher, between 5\% and $10 \%$. In selected trials that focused on homogenous MM patients, the incidence ranged between $3.2 \%$ and $10 \%$. Although the pathogenesis of $\mathrm{ONJ}$ is not understood, indirect evidence suggests that it is multifactorial and is clearly linked to long-term bisphosphonate use. Based on the biologic activity of bisphosphonates, ONJ is linked to adynamic bone, inhibition of angiogenesis, and microbial infections. ONJ typically occurs after dental procedures or local trauma, but it has also been shown to happen spontaneously. ${ }^{32,33}$

In the literature, the criteria used to diagnose ONJ have been inconsistent. Several associations and expert panels have provided consensus statements that include criteria for diagnosing ONJ; the details were recently reviewed. ${ }^{34}$ Bisphosphonaterelated $\mathrm{ONJ}$ can be defined as an area of exposed bone in the maxillofacial region that has not healed within 8 weeks after identification by a health care provider in a patient who is receiving or has been exposed to a bisphosphonate without local evidence of malignancy and no prior radiotherapy to the affected region. Patients may present with pain or feelings of numbness or heaviness of the jaw. Other symptoms include soft-tissue swelling, loosening of teeth, and infection. In many cases, $\mathrm{ONJ}$ is asymptomatic and is detected by the examining physician, who finds an exposed bone in the jaw. The mandible is the main affected site, and most lesions occur posterior to the cuspid teeth. . $^{33,35,36}$

In a recent longitudinal study documenting the natural history of ONJ, 96 patients from the United States and Greece were followed up for 3.9 years after ONJ diagnosis. ${ }^{35}$ Dental extractions preceded diagnosis of $\mathrm{ONJ}$ in $47 \%$ of cases, and these procedures were more common in patients with a single episode of $\mathrm{ONJ}$ than in those with recurrent and nonhealing disease ( $58 \%$ vs. $30 \%$ ). ONJ resolved in $62 \%$ of cases, recurred after healing in $12 \%$ (at the same or a new site) and did not resolve in $26 \%$ during 9 months of follow-up. The recurrence rate was higher among U.S. patients than Greek patients $(22 \%$ vs. $7 \%$, respectively). Discontinuation of bisphosphonate correlated with increased bone pain in the Greek cohort and increased fracture rates in the U.S. cohort; U.S. patients were more likely to restart bisphosphonate than were their Greek counterparts. Recurrence of ONJ was precipitated by re-initiation of bisphosphonate in 6 cases and dental treatment in 4 cases. The rate of MM relapse was higher in patients with recurrent or unresolved ONJ than in those who experienced a single such episode.

Management of $\mathrm{ONJ}$ is controversial because there are so many unknowns. No evidence-based consensus guidelines exist. A cornerstone of management is to attempt to prevent $\mathrm{ONJ}$ with good dental hygiene and avoidance of unnecessary dental procedures while on bisphosphonate therapy. 
For patients who do develop ONJ, a conservative, "supportive," nonsurgical approach has been recommended: chlorhexidine $0.12 \%$ oral rinses, intermittent systemic antibiotics, and careful sequestrectomy. Avoidance of bone curettage and surgical debridement is advised because most cases worsen after surgery. Ozone therapy, hyperbaric oxygen, and laser therapy have been used in several cases of $\mathrm{ONJ}$, with mixed results.

In an effort to decrease the occurrence of ONJ, in 2007 ASCO updated its guidelines on the use of bisphosphonate in patients with MM. ${ }^{37}$ The guidelines now recommend limiting the duration of bisphosphonate use to 2 years in patients with responsive or stable MM, with drug resumption recommended in the case of new-onset skeletal-related events. The guidelines also recommend that, before bisphosphonate initiation, patients should obtain a comprehensive dental examination, with particular attention to identifying active oral infections and sites at high risk for infection.

The incidence of $\mathrm{ONJ}$ may be lower in patients receiving 3-monthly bisphosphonate therapy versus monthly infusions ${ }^{38}$ however, the data are preliminary, and ongoing prospective clinical trials must be completed before such a schedule can be adopted.

\section{Renal Complications}

Renal impairment is common in patients with MM and may be present at diagnosis or a side effect of treatment. Bisphosphonates can cause renal function deterioration in the form of tubular necrosis and focal segmental glomerulosclerosis. If renal impairment is secondary to bisphosphonate use, discontinuation may lead to a partial recovery of function. ${ }^{39-41}$

The updated zoledronic acid prescribing information $^{42}$ and the updated ASCO guidelines ${ }^{37}$ recom- $^{-}$ mend a reduced dosage for patients with an estimated creatinine clearance between 30 and $60 \mathrm{~mL} / \mathrm{min}$. The dose should be administered over a period of no less than 15 minutes; administration time may be prolonged for up to 30 minutes to decrease risk of renal toxicity. ${ }^{42}$ Zoledronic acid is not recommended for patients with severe renal impairment (creatinine clearance $<30 \mathrm{~mL} / \mathrm{min}$ ). No dosing guidelines have been established for the use of pamidronate in patients with severe renal failure; however, ASCO recommends a reduced dosage. ${ }^{37}$ In general, bisphosphonates should be used with extreme caution in MM patients with renal impairment, and all MM patients should be monitored for deterioration of kidney function. ${ }^{3}$

Monitoring of creatinine levels during MM treatment is strongly recommended, although no current guidelines have been validated. Dosing adjustments are necessary for patients who exhibit a change in kidney function. ${ }^{3}$ There is currently no standard definition of renal failure, but most recent studies have used a serum creatinine level of $2 \mathrm{mg} / \mathrm{dL}$ as a cutoff point. Another useful marker is glomerular filtration rate, which has been suggested to be a more accurate measurement of renal function, especially for patients with mild or moderate renal impairment. ${ }^{43}$

\section{Anemia}

Anemia may be present at the time of MM diagnosis, or it may develop subsequently. The most common trigger is the replacement of bone marrow by plasma cells, but anemia can also result from factors related to MM treatment, such as chemotherapy, radiation

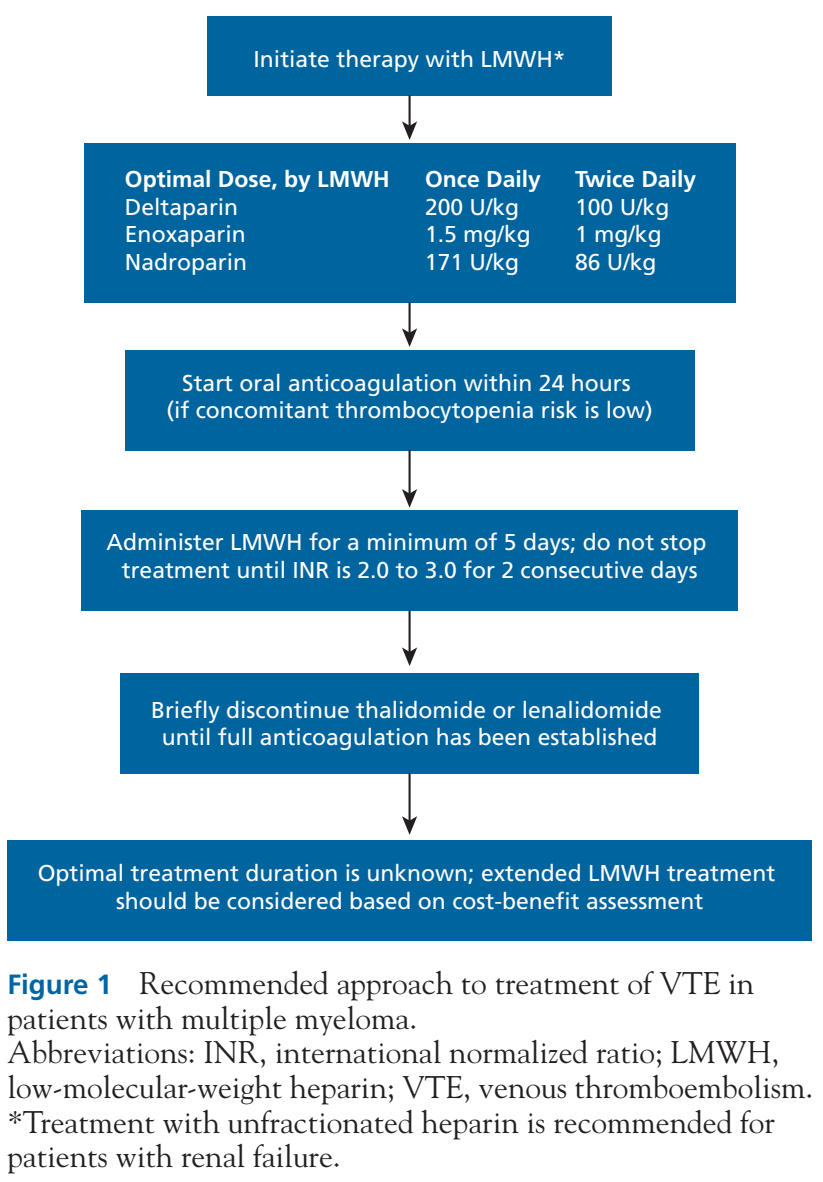


therapy, and $\mathrm{B}_{12}$ and folate deficiencies. ${ }^{44}$ Anemia often resolves when treatment for MM is swift and successful, but it will remain if the disease worsens.

Although anemia can be related to inadequate erythropoietin production, ${ }^{45}$ before initiating treatment, physicians should assess other possible causes. In particular, iron deficiency may be overlooked due to macrocytosis related to the treatment of MM. ${ }^{46}$ Patients with inadequate erythropoietin production may benefit from treatment with ESAs that mimic erythropoietin to stimulate red blood cell production. ${ }^{27-29}$ Treatment of anemia with ESAs has been successful in patients with a variety of cancer types, including MM. ${ }^{44}$ Studies specific to myeloma and lymphoma have shown improvement (response rates of $50 \%-70 \%$ ) in anemia-related outcomes, such as quality of life, hemoglobin levels, and number of required transfusions. ${ }^{45,47,48}$ If ESA use is not effective, the most likely causes are functional iron deficiency, infection, surgery, or plasma-cell bone marrow dysfunction. ${ }^{44}$

Unfortunately, recent evidence suggests that ESAs may contribute to solid tumor proliferation, thus reducing life expectancy. This was first noted in patients with breast or head and neck carcinoma. ${ }^{49}$ Research on ESA use in patients with MM has produced conflicting results. In a retrospective analysis, Baz et al..$^{50}$ identified a trend toward increased overall survival. A hazard ratio of $0.6(P=.026 ; 95 \% \mathrm{CI}$, 0.38-0.94) was identified for all patients except those with stage 1 disease, as defined by the Southwest Oncology Group staging system. No effect was found for patients with stage 1 disease. ${ }^{50}$ However, another retrospective analysis conducted by Katodrikou et al. ${ }^{51}$ found that ESAs might have a detrimental effect on both median overall survival and progression-free survival in patients with MM. Median overall survival was 31 months (95\% CI, 25-37 months) for patients receiving ESAs and 67 months (95\% CI, 55-79 months) for those not receiving ESAs. Median progression-free survival was 14 months (95\% CI, 12-16 months) for patients who received ESAs and 30 months (95\% CI, 24-36 months) for patients who did not $(P<.001$ for both comparisons). The authors concluded that their results do not contravene the therapeutic value of ESAs but might indicate that their administration should be limited to MM patients in whom a good response can be predicted. ${ }^{51}$ A randomized, prospective trial is needed to clarify the role and risk of ESAs in MM.

All ESAs are contraindicated for patients with uncontrolled hypertension. ${ }^{27-29}$ Revised FDA product labels for these drugs contain "black-box" warnings noting an increased risk of mortality, cardiovascular and thromboembolic events, and tumor progression or recurrence. In patients with renal failure, the FDA recommends individualized dosing with a goal of maintaining hemoglobin levels of 10 to $12 \mathrm{~g} / \mathrm{dL}$. In patients with cancer, ESAs are recommended for use only at the lowest doses and with concurrent chemotherapy. They are not recommended when myelosuppressive therapy is being used and a full recovery is expected. ${ }^{27-29}$ Based on these warnings, the NCCN recently released updated guidelines for the use of ESAs in cancer patients. ${ }^{52}$ The new guidelines eliminate hemoglobin level monitoring requirements and target hemoglobin ranges, reinforce the recommendation that only patients receiving chemotherapy should be treated with ESAs, and emphasize the risk for VTE with ESA administration.

For treatment with epoetin alfa, the FDA recommends administration of an anticoagulant prophylactic. ${ }^{27,28}$ Bortezomib combined with an ESA does not appear to be associated with increased VTE risk. ${ }^{30}$

\section{Conclusions}

The identification over the past 10 years of new, more effective primary treatments for MM, such as bortezomib, lenalidomide, and thalidomide, has led to meaningful changes in patient outcomes. However, lenalidomide and thalidomide, used either alone or in combination, are associated with elevated VTE risk. Likewise, the treatment of $\mathrm{MM}$-associated bony disease with bisphosphonate therapy provides clinical benefit but increases the risk for ONJ and renal impairment. Patients receiving MM therapy are also at heightened risk of anemia. The application of preventive measures, such as VTE prophylaxis, appropriate dental hygiene, and dose adjustments, can contribute to improved outcomes and quality of life for MM patients.

\section{Acknowledgment}

The authors thank Caitlin Rothermel, MA, for medical writing assistance. 


\section{References}

1. Geerts WH, Bergqvist D, Pineo GF, et al. Prevention of venous thromboembolism: American College of Chest Physicians evidence-based clinical practice guidelines (8th edition). Chest 2008;133(Suppl 6):S381-S453.

2. Heit JA, Silverstein MD, Mohr DN, et al. Risk factors for deep vein thrombosis and pulmonary embolism: a population-based case-control study. Arch Intern Med 2000;160:809-815.

3. Anderson KC, Alsina M, Bensinger W, et al. NCCN clinical practice guidelines in oncology: multiple myeloma, v 2.2010. Available at www.NCCN.org. Accessed August 12, 2009.

4. Brandenburg NA, Goss TF, Knight T, et al. Evaluation of the relationship between venous thromboembolism risk factors and the use of antithrombotic prophylaxis in multiple myeloma patients treated with thalidomide and dexamethasone combination regimens [abstract]. Blood 2008;112:Abstract 2369.

5. Zangari M, Elice F, Fink L, Tricot G. Thrombosis in multiple myeloma. Expert Rev Anticancer Ther 2007;7:307-315.

6. Rickles FR, Patierno S, Fernandez PM. Tissue factor, thrombin, and cancer. Chest 2003;124(Suppl 3):S58-S68.

7. Talamo GP, Ibrahim S, Claxton D, et al. Hypercoagulable states in patients with multiple myeloma can affect the thalidomideassociated venous thromboembolism. Blood Coagul Fibrinolysis 2009;20:337-339.

8. Niesvizky R, Martinez-Banos D, Jalbrzikowski J, et al. Prophylactic low-dose aspirin is effective antithrombotic therapy for combination treatments of thalidomide or lenalidomide in myeloma. Leuk Lymphoma 2007;48:2330-2337.

9. Coleman M, Vigliano EM, Weksler ME, Nachman RL. Inhibition of fibrin monomer polymerization by lambda myeloma globulins. Blood 1972;39:210-223.

10. Spicka I, Rihova Z, Kvasnicka J, et al. Disturbances of anticoagulation and fibrinolytic systems in monoclonal gammopathies-another mechanism of $\mathrm{M}$-protein interference with hemostasis. Thromb Res 2003;112:297-300.

11. Zangari $M$, Saghafifar $F$, Mehta $P$, et al. The blood coagulation mechanism in multiple myeloma. Semin Thromb Hemost 2003;29:275-282.

12. Palumbo A, Rajkumar SV, Dimopoulos MA, et al. Prevention of thalidomide- and lenalidomide-associated thrombosis in myeloma. Leukemia 2008;22:414-423.

13. Rajkumar SV, Rosinol L, Hussein M, et al. Multicenter, randomized, double-blind, placebo-controlled study of thalidomide plus dexamethasone compared with dexamethasone as initial therapy for newly diagnosed multiple myeloma. J Clin Oncol 2008;26:2171-2177.

14. Weber DM, Chen C, Niesvizky R, et al. Lenalidomide plus dexamethasone for relapsed multiple myeloma in North America. N Engl J Med 2007;357:2133-2142.

15. Gray KN, Chu D, Wu S, Lin RZ. Risk of venous thromboembolism with thalidomide in cancer patients: a systematic review and meta-analysis of randomized controlled trials [abstract]. Blood 2008;112:Abstract 3820.

16. Bennett CL, Angelotta C, Yarnold PR, et al. Thalidomide- and lenalidomide-associated thromboembolism among patients with cancer. JAMA 2006;296:2558-2560.

17. Sharma RA, Steward WP, Daines CA, et al. Toxicity profile of the immunomodulatory thalidomide analogue, lenalidomide: phase I clinical trial of three dosing schedules in patients with solid malignancies. Eur J Cancer 2006;42:2318-2325.
18. Niesvizky R, Jayabalan DS, Christos PJ, et al. BiRD (Biaxin [clarithromycin]/Revlimid [lenalidomide]/dexamethasone) combination therapy results in high complete- and overall-response rates in treatment-naive symptomatic multiple myeloma. Blood 2008;111:1101-1109.

19. Dimopoulos M, Spencer A, Attal M, et al. Lenalidomide plus dexamethasone for relapsed or refractory multiple myeloma. $\mathrm{N}$ Engl J Med 2007;357:2123-2132.

20. Boen M, McKoy J, West D, et al. Thalidomide (thal), lenalidomide (len), and dexamethasone (dex)-associated venous thromboembolism (VTE) and reported VTE rates pre- and postFDA approval: optimal prophylaxis strategies are still unclear [abstract]. Blood 2008;112:Abstract 2403.

21. Jacobus S, Kumar S, Callander NS, et al. Effect of venous thrombotic events on overall survival in multiple myeloma: analysis of thrombotic events occurring in E4A03A randomized trial of lenalidomide plus high-dose dexamethasone (RD) versus lenalidomide plus low-dose dexamethasone (Rd) in newly diagnosed multiple myeloma, a trial coordinated by the Eastern Cooperative Oncology Group (ECOG) [abstract]. Blood 2008;112: Abstract 1740.

22. Zangari M, Guerrero J, Cavallo F, et al. Hemostatic effects of bortezomib treatment in patients with relapsed or refractory multiple myeloma. Haematologica 2008;93:953-954.

23. Richardson PG, Sonneveld P, Schuster MW, et al. Bortezomib or high-dose dexamethasone for relapsed multiple myeloma. N Engl J Med 2005;352:2487-2498.

24. San Miguel JF, Schlag R, Khuageva NK, et al. Bortezomib plus melphalan and prednisone for initial treatment of multiple myeloma. N Engl J Med 2008;359:906-917.

25. Heit JA, Silverstein MD, Mohr DN, et al. Predictors of survival after deep vein thrombosis and pulmonary embolism: a populationbased, cohort study. Arch Intern Med 1999;159:445-453.

26. Bauer KA. The thrombophilias: well-defined risk factors with uncertain therapeutic implications [editorial]. Ann Intern Med 2001;135:367-373.

27. Epogen (epoetin alfa) for injection [package insert]. Thousand Oaks, CA: Amgen, Inc.; 2009.

28. Procrit (epoetin alfa) for injection [package insert]. Raritan, NJ: Ortho Biotech Products, L.P., 2009.

29. Aranesp (darbepoetin alfa) [package insert]. Thousand Oaks, CA: Amgen, Inc.; 2008.

30. Lonial S, Richardson PG, San Miguel J, et al. Characterisation of haematological profiles and low risk of thromboembolic events with bortezomib in patients with relapsed multiple myeloma. Br J Haematol 2008;143:222-229.

31. Bozlar U, Gaughen JR, Nambiar AP, Hagspiel KD. Imaging diagnosis of acute pulmonary embolism. Expert Rev Cardiovasc Ther 2007;5:519-529.

32. Badros A, Weikel D, Salama A, et al. Osteonecrosis of the jaw in multiple myeloma patients: clinical features and risk factors. J Clin Oncol 2006;24:945-952.

33. Migliorati CA, Casiglia J, Epstein J, et al. Managing the care of patients with bisphosphonate-associated osteonecrosis: an American Academy of Oral Medicine position paper. J Am Dent Assoc 2005;136:1658-1668.

34. Silverman SL, Landesberg R. Osteonecrosis of the jaw and the role of bisphosphonates: a critical review. Am J Med 2009;122(2 Suppl):S33-S45. 
35. Badros A, Terpos E, Katodritou E, et al. Natural history of osteonecrosis of the jaw in patients with multiple myeloma. J Clin Oncol 2008;26:5904-5909.

36. Mehrotra B, Ruggiero S. Bisphosphonate complications including osteonecrosis of the jaw. Hematology Am Soc Hematol Educ Program 2006:356-360, 515.

37. Kyle RA, Yee GC, Somerfield MR, et al. American Society of Clinical Oncology 2007 clinical practice guideline update on the role of bisphosphonates in multiple myeloma. J Clin Oncol 2007;25:2464-2472.

38. Corso A, Varettoni M, Zappasodi P, et al. A different schedule of zoledronic acid can reduce the risk of the osteonecrosis of the jaw in patients with multiple myeloma. Leukemia 2007;21:1545-1548.

39. Desikan R, Veksler Y, Raza S, et al. Nephrotic proteinuria associated with high-dose pamidronate in multiple myeloma. $\mathrm{Br} \mathrm{J}$ Haematol 2002;119:496-499.

40. Markowitz GS, Fine PL, Stack JI, et al. Toxic acute tubular necrosis following treatment with zoledronate (Zometa). Kidney Int 2003;64:281-289.

41. Markowitz GS, Perazella MA. Drug-induced renal failure: a focus on tubulointerstitial disease. Clin Chim Acta 2005;351:31-47.

42. Zometa (zoledronic acid) injection [package insert]. East Hanover, NJ: Novartis Pharmaceuticals Corp.; 2008.

43. Dimopoulos MA, Kastritis E, Rosinol L, et al. Pathogenesis and treatment of renal failure in multiple myeloma. Leukemia 2008;22:1485-1493.
44. Blade J, Rosinol L. Complications of multiple myeloma. Hematol Oncol Clin North Am 2007;21:1231-1246.

45. Mittelman M. The implications of anemia in multiple myeloma. Clin Lymphoma 2003;4(Suppl 1):S23-S29.

46. Ludwig H, Rai K, Blade J, et al. Management of disease-related anemia in patients with multiple myeloma or chronic lymphocytic leukemia: epoetin treatment recommendations. Hematol J 2002;3:121-130.

47. Birgegard G. Managing anemia in lymphoma and multiple myeloma. Ther Clin Risk Manag 2008;4:527-539.

48. Seidenfeld J, Piper M, Flamm C, et al. Epoetin treatment of anemia associated with cancer therapy: a systematic review and meta-analysis of controlled clinical trials. J Natl Cancer Inst 2001;93:1204-1214.

49. Lyman GH, Glaspy J. Are there clinical benefits with early erythropoietic intervention for chemotherapy-induced anemia? A systematic review. Cancer 2006;106:223-233.

50. Baz R, Walker E, Choueiri TK, et al. Recombinant human erythropoietin is associated with increased overall survival in patients with multiple myeloma. Acta Haematol 2007;117:162 167.

51. Katodritou E, Verrou E, Hadjiaggelidou C, et al. Erythropoiesisstimulating agents are associated with reduced survival in patients with multiple myeloma. Am J Hematol 2008;83:697-701.

52. Rogers GM, Becker PS, Bennett CL, et al. NCCN clinical practice guidelines in oncology: cancer- and chemotherapy-induced anemia, v 1.2009. Available at www.NCCN.org. Accessed August 12, 2009. 